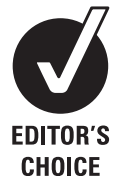

- An additional appendix is published online only. To view this files please visit the journal online (http://jnnp.bmj.com).

${ }^{1}$ Department of Psychosomatic Medicine and Psychotherapy, Klinikum rechts der Isar, Technische Universitaet Muenchen, Munich, Germany ${ }^{2}$ German Integrated Centre for Research and Treatment of Vertigo, Balance and Ocular Motor Disorders,

Ludwig-Maximilians-Universitaet, Klinikum Großhadern, Munich, Germany

${ }^{3}$ Department of Neurology, Ludwig-Maximilians-Universitaet, Klinikum Großhadern, Munich, Germany

\section{Correspondence to} Dr C Lahmann, Department of Psychosomatic Medicine and Psychotherapy, Klinikum rechts der Isar, Technische Universitaet Muenchen, Langerstr 381675 Munich, Germany; lahmann@tum.de

Received 31 August 2010 Revised 7 December 2010 Accepted 24 December 2010 Published Online First 4 February 2011

\title{
Psychotherapy in dizziness: a systematic review
}

\author{
G Schmid, ${ }^{1,2}$ P Henningsen, ${ }^{1,2}$ M Dieterich, ${ }^{2,3}$ H Sattel, ${ }^{1}$ C Lahmann ${ }^{1,2}$
}

\begin{abstract}
Background About $30-50 \%$ of complex dizziness disorders are organically not sufficiently explained or related to a psychiatric disorder. Of patients with such dizziness disorders, 80\% are severely impaired by dizziness in their daily and working lives; nevertheless, they are often not diagnosed or treated adequately.

Objectives This review aims to give a systematic overview of psychotherapeutic approaches and their efficacy regarding the treatment of dizziness that is medically not sufficiently explained or related to a psychiatric disorder.
\end{abstract}

Methods A systematic literature search was conducted in Medline, PSYNDEX and PsycINFO. Included in this systematic review were (randomised) controlled trials ((R)CTs) concerning psychotherapy in patients with dizziness, medically not sufficiently explained or associated with a psychiatric disorder. If possible, Hedges' g was used to express the effect sizes (ES) of the treatment. Heterogeneity was assessed using the 0 statistic. In addition, the quality of the studies was rated. Results Three (R)CTs were included. All studies used cognitive-behavioural treatment methods in combination with relaxation techniques or vestibular rehabilitation. All studies suggested that psychotherapy may provide improvement. The mean ES in the treatment groups was $0.46(95 \% \mathrm{Cl} 0.05$ to 0.88$)$ for dizziness related outcome, $0.10(-0.44$ to 0.64$)$ for anxiety and $0.17(-0.24$ to 0.58$)$ for depression whereas in the control groups the mean dizziness related ES was $-0.04(-0.44$ to 0.37$)$, anxiety related ES was $-0.03(-0.43$ to 0.38$)$ and depression related ES was $-0.02(-0.42$ to 0.38$)$. The quality of the studies was average. Sample sizes were small, however, and there was a lack of long term studies.

Conclusion This systematic review provides some preliminary evidence that psychotherapy may be effective in patients with dizziness that is medically not sufficiently explained or due to a psychiatric disorder. The results should be replicated in larger samples and follow-up RCTs.

\section{INTRODUCTION}

Dizziness and unsteadiness ${ }^{1}$ are common symptoms presented to general practitioners and neurologists. For individuals over the age of 75 years, these symptoms are the most frequent reason for visiting a physician. ${ }^{2}$ Their lifetime prevalence is about $20-30 \%$. $^{3}$ Approximately $30-50 \%$ of complex dizziness disorders (ie, dizziness is the cardinal symptom and persistent) are not fully explained by an identifiable medical illness and are related to phobic, panic, anxiety, depressive, dissociative or somatoform disorders. ${ }^{4-7}$ Dizziness can occur without a prior organic vestibular disorder or as a consequence of an organic vestibular disorder, particularly in patients with a vestibular migraine or
Menière's disease. ${ }^{8}$ About $30 \%$ of all patients with peripheral vestibular vertigo subsequently develop dizziness or unsteadiness that is not fully medically explained or related to a psychiatric disorder. ${ }^{5}$ Godemann and colleagues ${ }^{9}$ found that a lack of social support, a high burden of suffering and moderate to severe impairment of self-experience were associated with the development of a panic disorder after an episode of an acute vestibular disorder. Phobic postural vertigo (PPV) frequently follows a period of an organic vestibular disorder, a serious illness or emotional stress, ${ }^{10}$ and is characterised by a combination of non-rotational vertigo with subjective postural and gait instability. ${ }^{11}$ It is often associated with anxiety, is context dependent (eg, occurs in large crowds) and results in avoidance behaviour. The symptoms improve when the patient has taken a small amount of alcohol or engages in sports. ${ }^{12}$

There seems to be a link between anxiety and balance disorders ${ }^{13}$ : patients with panic or anxiety and with vestibular disorders frequently present with similar behaviour-namely, avoidance and similar complaints (ie, dizziness, spatial disorientation and anxiety in particular environments). ${ }^{14}$ Additionally, increased visual dependence (ie, subjects who preferentially use vision for spatial orientation and postural control) and thus increased body sway can be observed both in patients with primary vestibular disorders and in patients with anxiety disorders. ${ }^{15} 16$ Therefore, the hypothesis was discussed that vestibular or balance dysfunctions occur particularly in panic patients with moderate to severe agoraphobia. ${ }^{7}$

About $80 \%$ of patients with dizziness that is medically not sufficiently explained or related to a psychiatric illness feel impaired in their daily and working lives or are even unable to work due to the dizziness. ${ }^{5}$ Because of this substantial impact of dizziness on daily functioning, an adequate intervention is necessary. However, these patients often undergo an odyssey of visits to different physicians until the correct diagnosis is made and an appropriate treatment can be administered. The therapy has to include a discussion on a psychosomatic illness concept and a demonstration of associations between organic and psychological states and sensations. Mild forms can be treated with short time interventions ${ }^{17}$ whereas complex dizziness syndromes require specific interdisciplinary and integrative treatment that includes physiotherapy (eg, vestibular rehabilitation), pharmacotherapy and psychotherapy. 5121819

\section{Dizziness specific treatment requirements}

To determine the appropriate management of dizziness and unsteadiness, the patient has to be asked how far dizziness affects his or her life. ${ }^{2}$ For 
example, some patients may have no unsteadiness while walking but can no longer play golf or tennis due to their imbalance. These patients require limited vestibular rehabilitation, while patients severely affected by their dizziness (ie, they are unable to leave their house or go to work) need extensive counselling and physical therapy. In the following paragraphs, some dizziness specific treatment elements are described.

\section{Vestibular rehabilitation}

Vestibular rehabilitation (VR) has been recommended as the treatment of choice for patients with persistent vertigo due to vestibular dysfunction. The central element of VR is a set of exercises that promote central compensation by providing the CNS with repeated exposure to a range of eye, head and body movements that require the patient's compliance and active collaboration. ${ }^{20-22}$ This approach aims at the habituation or remediation of dizziness, improvement of gaze, retraining of balance and enhancement of physical fitness. The mechanisms of balance and vestibular compensation are explained to the patient in detail so that the patient can understand why physiotherapy is reasonable. ${ }^{22}$ In controlled studies, VR has been shown to be efficient in patients suffering from vertigo and balance disorders. ${ }^{23}$ Furthermore, VR has positively influenced the emotional condition of patients with chronic vestibular deficits $^{24}$ and improved both the independence in daily living activities $^{25}$ and the patient's quality of life. ${ }^{26}$

\section{Cognitive-behavioural approaches}

The cognitive-behavioural approach aims to develop an integrative explanation model for dizziness taking into account analysis, the first dizziness attack, in particular, has to be explored extensively. Additionally, factors that trigger, sustain or intensify the dizziness have to be analysed and evaluated-for example, irrational cognitions. ${ }^{28}$ In vivo exposures to dizziness triggering stimuli and exercises form part of the treatment. ${ }^{29}$ In terms of exposure procedures, cognitive-behavioural approaches are similar to VR. Moreover, relaxation techniques are taught, and effects of visuovestibular mismatch. ${ }^{30}$ Furthermore, patients suffering from dizziness related to a psychiatric disorder increasingly activate their posture musculature due to anxiety and thus show a reduced horizontal and an increased vertical sway path that can be normalised within a short term behavioural therapy. ${ }^{31}$

\section{Aim of this systematic review}

In summary, the psychotherapeutic treatment of dizziness that is medically not sufficiently explained or due to phobic, panic, anxiety, depressive, dissociative or somatoform disorders has been, to date, subject to expert opinion review only; there is a lack of systematic reviews of controlled trials or evidence based guidelines. Thus we wished to give an overview of the psychotherapeutic approaches that are available for dizziness and their efficacy in the form of a systematic review considering all (randomised) controlled trials ((R)CTs) obtainable at present. This can be seen as a first important step towards identifying future directions and to work out guidelines for treating dizziness that is medically not sufficiently explained or related to a psychiatric illness, and-if applicable-to develop a therapy manual for these dizziness disorders.

\section{METHODS}

Relevant articles were identified by searching Medline, PSYNDEX and PsycINFO from 1900 to June 2010. The medical and psychological factors. ${ }^{27}$ With the use of behaviour there is a focus on how the person can minimise the disorienting

following keywords were searched: dizziness, vertigo, Menière, vestibular migraine, benign paroxysmal positional and vestibular neuritis. These terms were cross referenced with the following keywords: controlled trial, psychotherapy, psychotherapeutic treatment, intervention and behaviour therapy. In addition, to ensure a comprehensive review of the literature, we searched for further relevant citations by checking the reference lists of the initial studies identified and of review papers. This searching strategy revealed more than 1000 papers (see figure 1).

Studies were included if they were in English or German; if they had been conducted on humans suffering from dizziness, medically not sufficiently explained or associated with phobic, panic, anxiety, depressive, dissociative or somatoform disorders, or persistent dizziness after an organically explained condition; if the therapy described was a psychotherapy (eg, a cognitivebehavioural therapy (CBT)); if the paper was not a review paper with expert opinions; and if the study design was an (R)CT. With the use of that strategy, up to the end of June 2010, a total of four original papers (or three studies) that met the inclusion criteria emerged (see figure 1). ${ }^{17} 32-34$ Literature research and selection was carried out independently by two researchers (GS and $\mathrm{CL}$ ). In addition, inclusion of studies was discussed between GS and CL. Two conference papers ${ }^{31} 35$ were not included in this review as the data reported were not detailed enough to compute effect sizes and further data were not available from the authors as the data have not yet been published.

To evaluate the efficacy of the interventions, effect sizes (ES; Hedges' g) and 95\% confidence intervals (95\% CI) were calculated concerning dizziness, anxiety and depression related outcomes (see figure 2A-C). Hedges' g statistic was computed as this is an estimate of the standardised mean difference that is bias corrected for small sample sizes. ${ }^{36}$ The ES was interpreted as follows: small effect if $0.2 \leq|\mathrm{ES}|<0.5$, medium effect if $0.5 \leq 1$

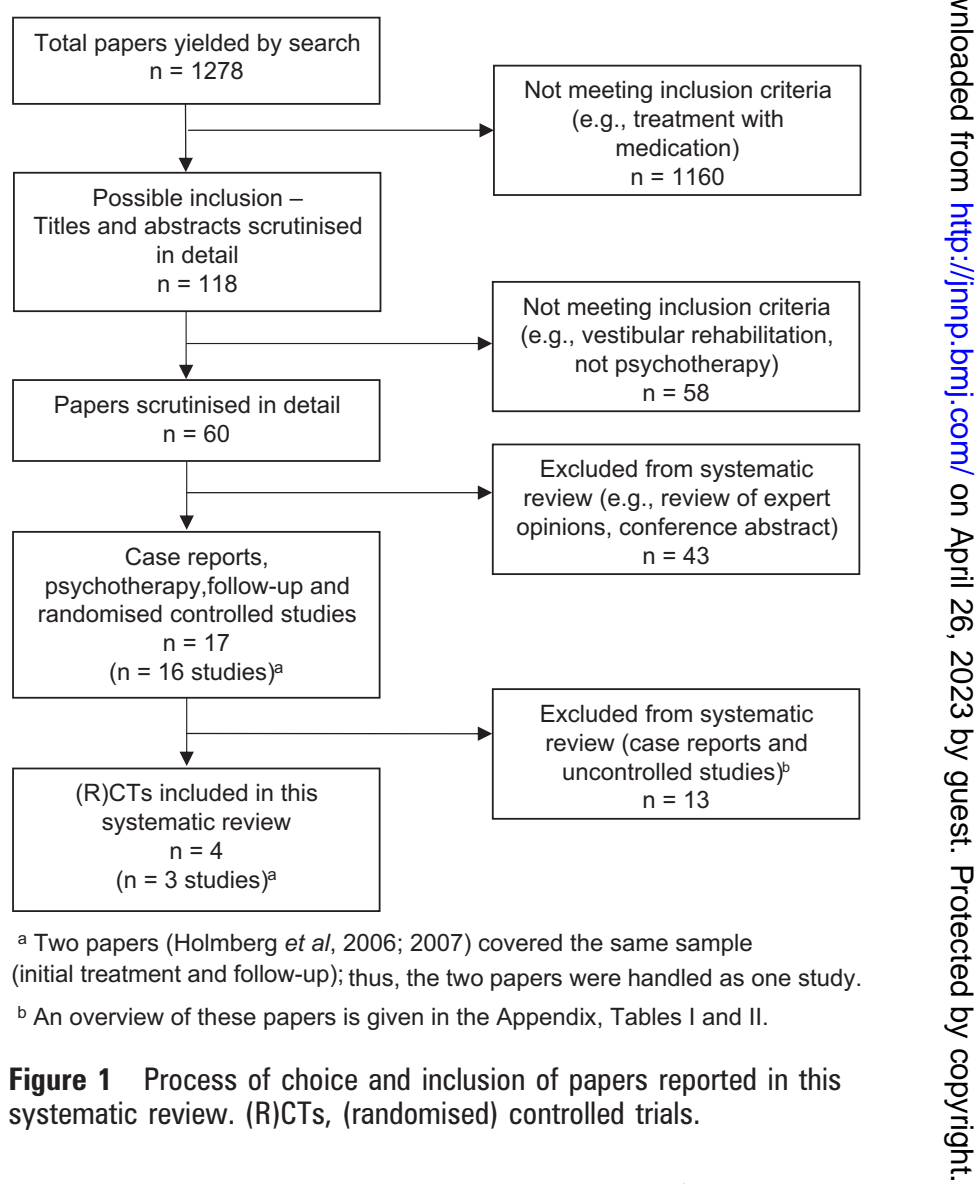


Figure 2 (A) Dizziness related effect sizes of (randomised) controlled trials. (B) Anxiety related effect sizes of (randomised) controlled trials.

(C) Depression related effect sizes of (randomised) controlled trials.

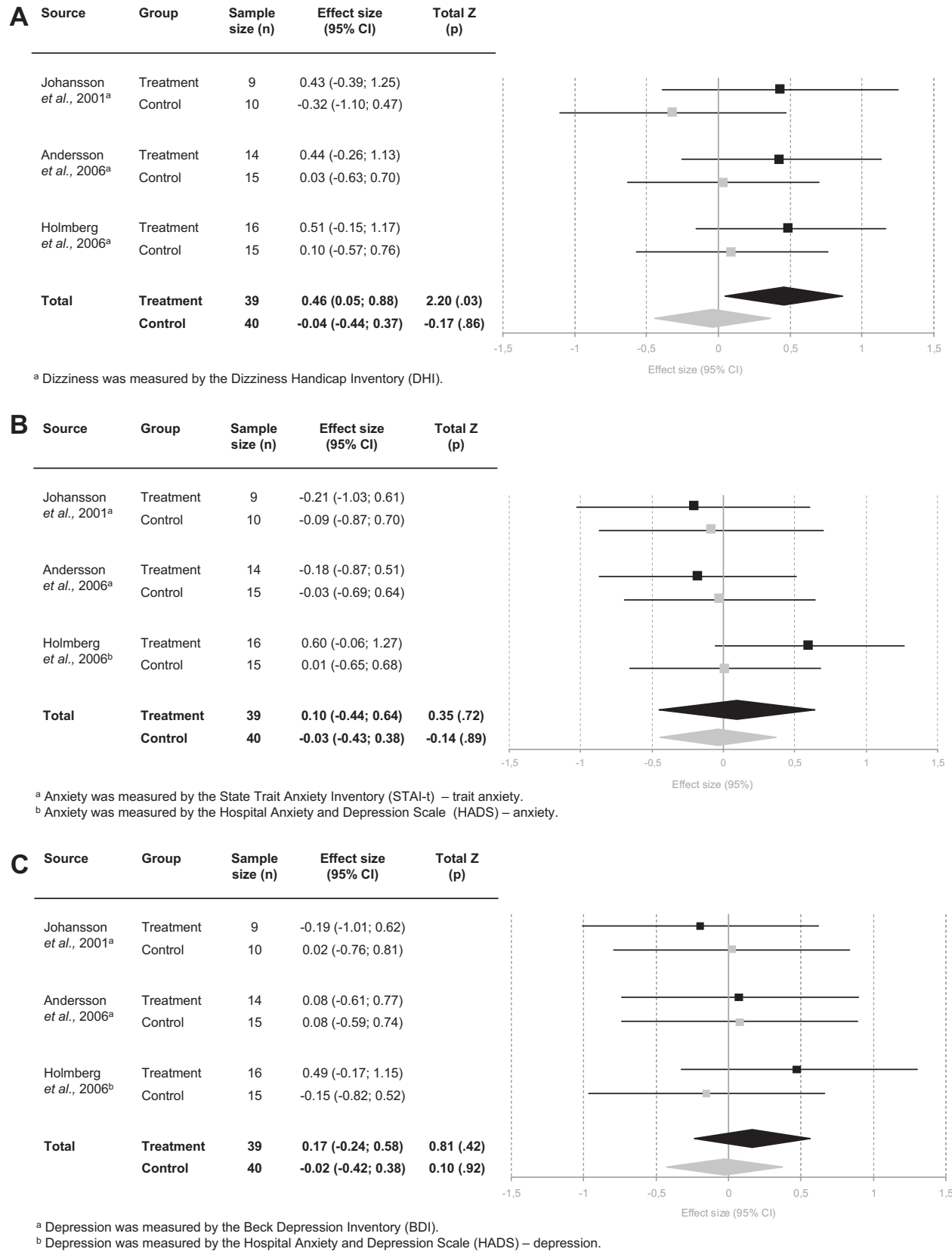

b Depression was measured by the Hospital Anxiety and Depression Scale (HADS) - depression.
ES $\mid<0.8$ and large effect if $|E S| \geq 0.8$. $^{37}$ To ensure an appropriate interpretation of the results, the $Z$ statistic (test of null) was estimated and the $p$ value was set at $<0.05$. Furthermore, tests for heterogeneity were carried out using the $Q$ statistic $(p<0.05)$ to evaluate whether the variation in study outcomes between studies was significant. ${ }^{38}$ Statistical analyses were conducted using Comprehensive Meta-Analysis V.2. ${ }^{39}$

Furthermore, GS and a research assistant independently rated the quality of all reviewed studies using the Cochrane Collaboration Depression, Anxiety and Neurosis (CCDAN) quality assessment rating scale by Moncrieff and colleagues. ${ }^{40}$ The 23 items were rated on a three point scale $(0=$ criterion not fulfilled, $1=$ half fulfilled, $2=$ fulfilled) and assessed topics such as objectives and specification of main outcomes, adequacy of sample size, clear description of treatments, recording of exclusion criteria, report of number of exclusions and refusals, sample demographics, clear description of outcome measures or use of validated instruments, appropriateness of statistical analyses and conclusions. Table 1 reports the mean quality assessment rating scores by GS and the research assistant and the proportions for each study.

\section{RESULTS}

Table 1 summarises the (R)CTs. An overview of the case reports and uncontrolled studies is given in tables I and II in the appendix (available online only).

\section{Patients}

The studies reviewed included a total of 87 patients $(60$ women and 27 men) with a range over the studies of 22-36 patients. Patients suffered from PPV, ${ }^{10}$ vestibular dysfunction, tension related medically unexplained dizziness, previous diagnosis of Menière's disease, whiplash or neck related problems, or mixed problems. They were either recruited via newspaper advertisements or were referred by neurotological specialists. Table 1 


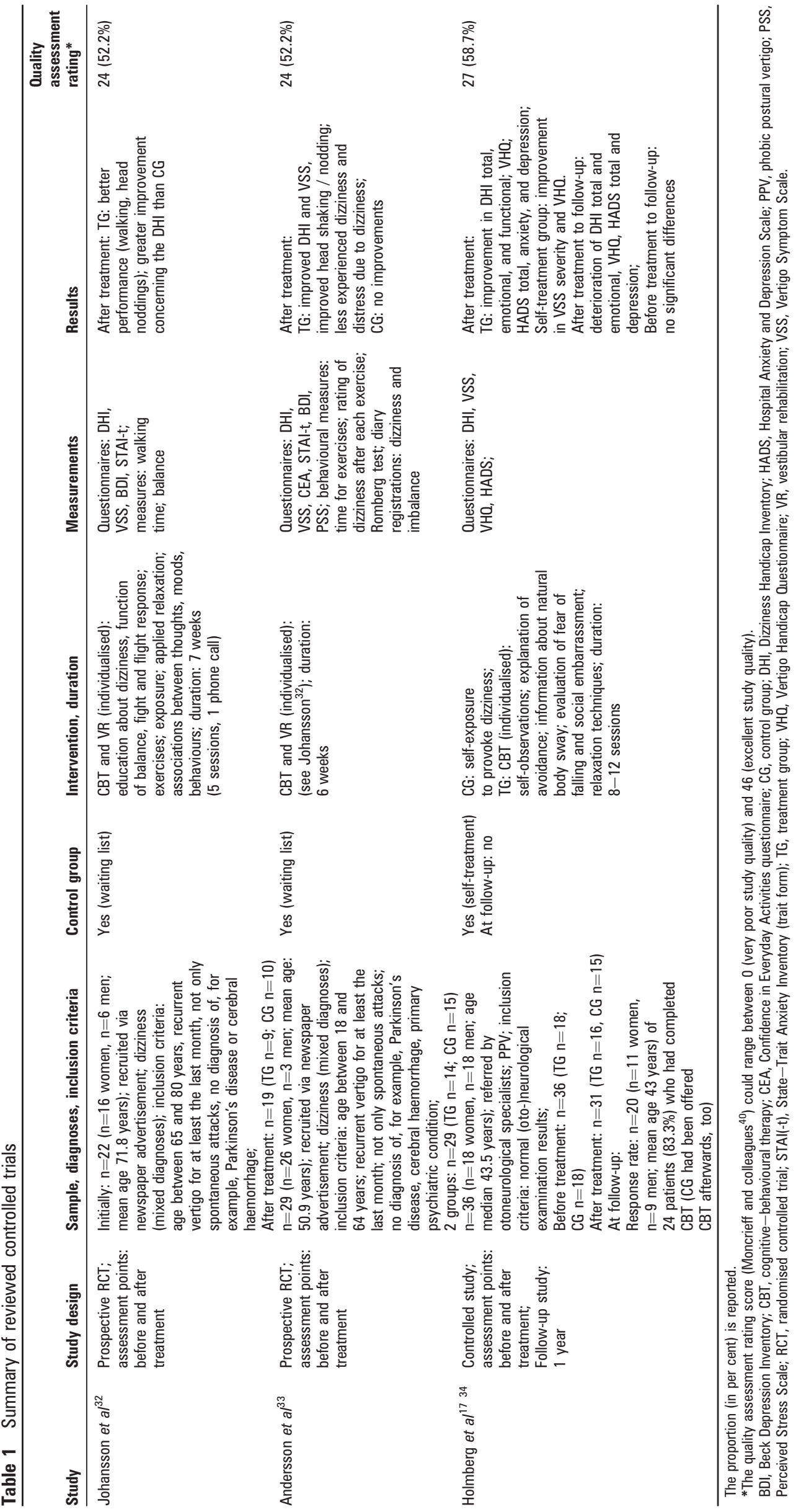


shows the inclusion criteria for each study. The duration of treatment ranged from 5 to 12 sessions (see table 1).

\section{Treatment approaches and measures}

All studies used cognitive-behavioural treatment methods, combined with relaxation techniques, and/or VR, and consisted of the following elements: information and education about dizziness and the balance and vestibular system; explanation and discussion of associations between assumptions (about dizziness), arousal, thoughts, moods and behaviours; (selfcontrolled) exposure to fear triggering and avoidant situations; coping strategies; balance exercises; and self-observations and written recordings. In two studies CBT was combined with VR. The purpose of providing VR within a CBT framework is to facilitate exposure to movements and to teach strategies to cope with associated thoughts and beliefs concerning the dizziness.

The studies provided as outcome measures dizziness related factors (ie, severity, frequency, handicap and distress due to dizziness, imbalance, walking time) and, in addition, depression and anxiety were assessed. For measuring treatment outcome, all studies used standardised questionnaires (eg, the Dizziness Handicap Inventory ${ }^{41}$ or the Beck Depression Inventory) ${ }^{42}$ (see table 1).

\section{Treatment outcomes}

All studies suggest that psychotherapy (ie, cognitivebehavioural interventions combined with VR or relaxation techniques) provides improvement in patients suffering from dizziness (eg, PPV, medically unexplained, previous Menière's disease) (see table 1). However, only one study conducted a follow-up and found no long term effect: at the 1 year followup, the test results were similar to those obtained before treatment, indicating that the CBT effects were not persistent in patients with $\mathrm{PPV}^{34}$

\section{Efficacy of treatments}

The ES were divided into three outcome domains: dizziness, anxiety and depression related (see figure $2 \mathrm{~A}-\mathrm{C}$ ).

\section{Tests for heterogeneity}

Neither in the treatment nor in the control groups were the tests for heterogeneity significant concerning dizziness $\left(\mathrm{O}_{\text {treatment }}=0.03, \quad \mathrm{df}_{\text {treatment }}=2, \mathrm{p}_{\text {treatment }}=0.99 ; \mathrm{Q}_{\text {control }}=0.68\right.$, $\left.\mathrm{df}_{\text {control }}=2, \mathrm{p}_{\text {control }}=0.71\right)$, anxiety $\left(\mathrm{O}_{\text {treatment }}=3.40, \mathrm{df}_{\text {treatment }}=2\right.$, $\left.p_{\text {treatment }}=0.18 ; \quad Q_{\text {control }}=0.04, \quad \mathrm{df}_{\text {control }}=2, \quad p_{\text {control }}=0.98\right)$ or depression $\quad\left(\mathrm{Q}_{\text {treatment }}=1.72, \quad \mathrm{df}_{\text {treatment }}=2, \quad p_{\text {treatment }}=0.42\right.$; $\mathrm{O}_{\text {control }}=0.24, \mathrm{df}_{\text {control }}=2, \mathrm{p}_{\text {control }}=0.89$ ), indicating that there were no significant variations in study outcomes between studies.

\section{Efficacy of treatment and control groups}

Both Johansson and colleagues ${ }^{32}$ and Andersson and colleagues ${ }^{33}$ reported small but clinically not relevant effects concerning dizziness, and a small but not significant deterioration regarding anxiety and depression with their combined CBT and VR approach. In contrast, Holmberg and colleagues, ${ }^{17}$ who conducted CBT, achieved medium but not significant effects with respect to dizziness and anxiety and an almost medium but clinically not relevant effect concerning depression (see figures $2 \mathrm{~A}$ and 2C). At follow-up, 1 year after treatment, however, none of the patients were symptom free and test results were similar to those before treatment. ${ }^{34}$ Overall, the three CBT approaches reached a small and clinically relevant effect concerning dizziness and no effects in terms of anxiety and depression (see figures $2 \mathrm{~A}$ and $2 \mathrm{C}$ ).

There were no effects in the control groups (waiting list or self-treatment), nor were the overall control group effects significant in terms of dizziness, anxiety or depression (see figures $2 \mathrm{~A}$ and $2 \mathrm{C}$ ).

Concerning dizziness, the ES difference between the treatment and control groups was $|0.5|$. With 40 or 39 persons in each group, the power to detect differences would be 0.60 and thus it would be doubtful to prove statistically significant group differences. ${ }^{43}$

\section{Quality assessment rating of studies}

The quality score of the studies ranged between 24 and 27, which corresponds to $52.2 \%$ and $58.7 \%$ of the 46 possible points of the CCDAN quality assessment rating scale (see table 1).

\section{DISCUSSION}

This systematic review aimed to give an overview of psychotherapy approaches concerning dizziness and their efficacy. In summary, all (R)CTs used CBT approaches. Two of these studies used a combination of CBT and VR. All studies reported an improvement after treatment, particularly in terms of dizziness. However, all studies had methodological shortcomings, such as small sample sizes and/or lack of follow-up.

As the tests for heterogeneity were not significant, one can assume that the ES of the different studies were homogeneous. ${ }^{38}$ Furthermore, the studies were predominantly uniform regarding sample sizes, treatment methods and duration of treatments. In addition, all studies measured dizziness, anxiety and depression before and after treatment using standardised questionnaires. Thus the results and outcome measures may be comparable and the studies can be considered together. However, the patients in the study of Johansson and colleagues ${ }^{32}$ were much older than the patients in the two other studies. ${ }^{17} 33$ Additionally, the recruitment methods differed (see table 1), which may have led to a sample bias (ie, the participants may not be representative but especially impaired or motivated). Finally, the level of impairment was not specified or determined which might have caused a result bias. Thus the results should be replicated with representative and larger samples.

All three studies together reached small but significant ES in terms of dizziness. The unpublished and preliminary results of Best and colleagues ${ }^{31}$ and Tschan and colleagues ${ }^{35}$ support the hypothesis that short term CBT may be effective-even in the long term -in normalising the patients' body posture, in reducing the severity of dizziness symptoms and in modifying dysfunctional illness experiences. However, Holmberg and colleagues ${ }^{17} 34$ found no significant difference between the psychometric test results before treatment and 1 year later, and none of the patients was symptom free at follow-up. ${ }^{34}$ Holmberg et al argued that this result confirms the observation that the treatment of PPV is complex, and that their CBT method, which was developed during their project, needs further attention and might lead to better results in a manualised intervention.

Both Johansson and colleagues ${ }^{32}$ and Andersson and colleagues, ${ }^{33}$ who conducted a combination of CBT and VR, showed in RCTs small but clinically not relevant ES concerning dizziness in patients with mixed dizziness diagnoses and a broad age range. Combining CBT and VR seems to be reasonable as VR programmes already cover implicitly some psychotherapeutic elements (ie, both approaches address the functional impairment due to the disorder, develop an individualised treatment plan and provide exposure to reach habituation). In addition, it has been shown that a combination of VR and a 30 min therapy session improved postural control, subjective symptom report and emotional well being, even in the long term. ${ }^{44}$ Yardley and colleagues $^{45}$ found that exercises that stimulate the vestibular system and provoke dizziness can modify negative beliefs about 
the consequences of dizziness. Patients with both panic disorder and vestibular dysfunctions may benefit in particular from the combination of CBT and VR. ${ }^{14}$ In further research, the efficacy of the CBT/VR approach should be tested against other interventions, with larger samples, and in the long term course.

However, Johansson and colleagues, ${ }^{32}$ Andersson and colleagues $^{33}$ and Holmberg and colleagues ${ }^{17}$ did not achieve any clinically relevant improvement concerning anxiety or depression. This indicates that the CBT treatment approach (in combination with VR) should be expanded to address these issues (eg, by focusing on anxiety symptoms other than dizziness, particularly cognitive symptoms). ${ }^{46}$ To enhance existing treatment programmes it may be reasonable to offer a basic psychotherapeutic module to all patients suffering from dizziness and unsteadiness. This basic treatment should consist of education and information about the natural body sway, unsteadiness, dizziness and the function of balance, exercises to improve balance and exposure to dizziness triggering situations. Additionally, specific treatment modules tailored for patients with depression, anxiety, phobic, panic or somatoform related dizziness should be offered. For example, patients with somatoform related dizziness should participate in the somatoform module consisting of enhancement of bodily activities, improving the distinction between bodily complaints and effects, and effect regulation. The multicentre PISO study currently evaluates a new three phased short term psychodynamic intervention that is tailored for pain predominant somatoform disorder. $^{47} 48$ This therapy approach may also be adequate for patients with dizziness related to a somatoform disorder.

In summary, the present systematic review provides some preliminary evidence that psychotherapy may be effective in patients suffering from dizziness, such as PPV, medically unexplained or a previous Menière's disease. More importantly, however, it reveals a lack of (R)CTs and long term follow-up studies. In addition, the results are not generalisable as the samples were not representative and sample sizes were very small. In further research, the efficacy of psychotherapy in dizziness should be replicated applying RCTs with larger, representative samples and standardised psychotherapy manuals that could be developed in light of the results of this review.

Funding This project was supported by funds from the German Federal Ministry of Education and Research under the grant code 01 E0 0901. The authors bear full responsibility for the content of this publication.

Competing interests None.

Provenance and peer review Not commissioned; externally peer reviewed.

\section{REFERENCES}

1. Bisdorff A, Von Brevern M, Lempert T, et al. Classification of vestibular symptoms: towards an international classification of vestibular disorders. J Vestib Res 2009;19:1-13.

2. Tusa RJ. Dizziness. Med Clin North Am 2009;93:263-71, vii.

3. Neuhauser HK. Epidemiology of vertigo. Curr Opin Neurol 2007;20:40-6.

4. Dieterich M, Eckhardt-Henn A. Neurological and somatoform vertigo syndromes. Nervenarzt 2004:75:281-302.

5. Eckhardt-Henn A, Tschan R, Best C, et al. Somatoform vertigo syndrome Nervenarzt 2009;80:909-17.

6. Ronel J, Lahmann C. Vertigo and psyche. Nervenheilkunde 2008-27:846-52.

7. Furman JM, Jacob RG. Psychiatric dizziness. Neurology 1997:48:1161-6.

8. Eckhardt-Henn A, Best C, Bense S, et al. Psychiatric comorbidity in different organic vertigo syndromes. J Neurol 2008:255:420-8.

9. Godemann F, Schuller J, Uhlemann H, et al. Psychodynamic vulnerability factors in the development of panic disorders - a prospective trial in patients after vestibular neuritis. Psychopathology 2009:42:99-107.

10. Brandt T. Phobic postural vertigo. Neurology 1996;46:1515-19.

11. Brandt T, Huppert D, Dieterich M. Phobic postural vertigo: A first follow-up. J Neurol 1994:241:191-5.

12. Strupp M, Brandt T. Diagnosis and treatment of vertigo and dizziness. Dtsch Arzteb/ Int 2008:105:173-80.

13. Furman JM, Balaban CD, Jacob RG, et al. Migraine-anxiety related dizziness (MARD): a new disorder? J Neurol Neurosurg Psychiatry 2005:76:1-8.
14. Beidel DC, Horak FB. Behavior therapy for vestibular rehabilitation. J Anxiety Disord 2001:15:121-30

15. Jacob RG, Redfern MS, Furman JM. Space and motion discomfort and abnormal balance control in patients with anxiety disorders. J Neurol Neurosurg Psychiatry 2009;80:74-8

16. Bronstein AM. Under-rated neuro-otological symptoms: Hoffman and Brookler 1978 revisited. Br Med Bull 2002;63:213-21.

17. Holmberg J, Karlberg M, Harlacher U, et al. Treatment of phobic postural vertigo. A controlled study of cognitive-behavioural therapy and self-controlled desensitization. J Neurol 2006;253:500-6.

18. Bronstein AM, Lempert T. Management of the patient with chronic dizziness. Restor Neurol Neurosci 2010:28:83-90.

19. Henningsen $\mathbf{P}$, Zipfel S, Herzog W. Management of functional somatic syndromes Lancet 2007;369:946-55.

20. Telian SA, Shepard NT. Update on vestibular rehabilitation. Otolaryngol Clin North Am 1996;29:359-71.

21. Luxon LM. Handbook of vestibular rehabilitation. London: Whurr Publishers Ltd, 1997.

22. Luxon LM. Evaluation and management of the dizzy patient. J Neurol Neurosurg Psychiatry 2004;75(Suppl IV):iv45-52

23. Boyer FC, Percebois-Macadré L, Regrain E, et al. Vestibular rehabilitation therapy. Neurophysiol Clin 2008;38:479-87

24. Meli A, Zimatore G, Badaracco C, et al. Effects of vestibular rehabilitation therapy on emotional aspects in chronic vestibular patients. J Psychosom Res 2007:63:185-90

25. Cohen HS, Kimball KT. Increased independence and decreased vertigo after vestibular rehabilitation. Otolaryngol Head Neck Surg 2003:128:60-70.

26. Badaracco C, Labini FS, Meli A, et al. Vestibular rehabilitation outcomes in chronic vertiginous patients through computerized dynamic visual acuity and Gaze stabilization test. Otol Neurotol 2007;28:809-13.

27. Langs G. Behavioural oriented medical aspects of vertigo. Psychoneuro 2004:30:317-21.

28. Schaaf H. Psychotherapy in vertigo disorders, 2nd Edn. Kröning: Asanger Verlag $\mathrm{GmbH}, 2007$.

29. Schaaf H. Behavioural therapy approaches in vertigo disorders. Psychomed 1998;10:88-92.

30. Asmundson GJG, Larsen DK, Stein MB. Panic disorder and vestibular disturbance: an overview of empirical findings and clinical implications. J Psychosom Res 1998:44:107-20

31. Best C, Tschan R, Dellani P, et al. Improved postural control after behavioural short-term intervention in patients with psychiatric dizziness. Akt Neurologie 2008;35(Suppl 1):S117-18.

32. Johansson $\mathbf{M}$, Akerlund $\mathrm{D}$, Larsen $\mathrm{HC}$, et al. Randomized controlled trial of vestibular rehabilitation combined with cognitive-behavioral therapy for dizziness in older people. Otolaryngol Head Neck Surg 2001:125:151-6.

33. Andersson G, Asmundson GJG, Denev J, et al. A controlled trial of cognitive-behavior therapy combined with vestibular rehabilitation in the treatmen of dizziness. Behav Res Ther 2006;44:1265-73.

34. Holmberg J, Karlberg M, Harlacher U, et al. One-year follow-up of cognitive behavioral therapy for phobic postural vertigo. J Neurol 2007;254:1189-92.

35. Tschan R. Scheurich V, Best C, et al. Manualized short-term intervention for patients with somatoform vertigo syndromes. 60. Arbeitstagung des Deutschen Kollegiums für Psychosomatische Medizin (DKPM) und 17. Jahrestagung der Deutschen Gesellschaft für Psychosomatische Medizin und Ärztliche Psychotherapie (DGPM). Mainz, 2009.

36. Hedges LV. Distrubution theory for Glass's estimatory effect size and related estimators. J Educ Stat 1981:6:107-28.

37. Cohen J. Statistical power analysis for the behavioural sciences. New Jersey: Lawrence Erlbaum Associates, 1998.

38. Borenstein M, Hedges LV, Higgins JPT, et al. Introduction to meta-analysis. West Sussex: John Wiley \& Sons Ltd, 2009.

39. Borenstein M, Hedges LV, Higgins JPT, et al. Comprehensive meta-analysis version 2. Englewood NJ: Biostat, 2005.

40. Moncrieff J, Churchill R, Drummond DC, et al. Development of a quality assessment instrument for trials of treatment for depression and neurosis. Int $J$ Methods Psychiatr Res 2001;10:126-33.

41. Jacobson GP, Newman CW. The development of the Dizziness Handicap Inventory Arch Otolaryngol Head Neck Surg 1990;116:424-7.

42. Beck AT, Steer RA. Beck Depression Inventory Manual. San Antonio, TX: The Psychological Corporation, 1987.

43. Faul F, Erdfelder E, Lang AG, et al. G*Power 3: a flexible statistical power analysis program for the social, behavioral, and biomedical sciences. Behav Res Methods 2007;39:175-91.

44. Yardley L, Beech S, Zander L, et al. A randomized controlled trial of exercise therapy for dizziness and vertigo in primary care. Br J Gen Pract 1998;48:1136-40.

45. Yardley L, Beech S, Weinman J. Influence of beliefs about the consequences of dizziness on handicap in people with dizziness, and the effect of therapy on beliefs. J Psychosom Res 2001;50:1-6.

46. Taylor S, Asmundson GJG. Treating health anxiety: a cognitive-behavioural approach. New York: Guilford Press, 2004

47. Lahmann C, Henningsen P, Noll-Hussong M, et al. Somatoform disorders. Psychother Psychosom Med Psychol 2010;60:227-33.

48. Lahmann C, Sack M, Ronel J. PISO — an evidence-based approach in the therapy of somatoform disorders. PDP 2007;6:131-9. 